\title{
>SO WIRD ES SCHLIESSLICH DEIN BILD SEIN, DAS FÜR DICH TANZT<. \\ THEORIEGESCHICHTLICHE KONZEPTE EINER INTERART-POETIK VON FILM UND TANZ
}

\author{
KRISTINA KÖHLER
}

Dass künstlerischer Tanz im 20. Jahrhundert längst nicht mehr nur im Theaterdispositiv oder in vergleichbaren theatralen Aufführungszonen stattfindet, sondern dass Medien und insbesondere Medien bewegter Bilder zu einem Ort des Tanzes werden können, verhandeln Film- und Tanztheoretiker spätestens seit der Erfindung des Kinematographen. Während die Filmwissenschaften Tanz zumeist als Motiv >im< Film hinsichtlich seiner symbolischen Bedeutung für die narrative Ebene untersuchen (vgl. z.B. Krah 2003), verhandelt der Diskurs von Tänzern, Choreographen und Tanztheoretikern wiederum vornehmlich den Mediensprung vom leibhaftigen, präsentischen Bühnentanz zum reproduzierten, technischen Bewegungsbild (vgl. z.B. Jordan/Allen 1993; Brooks/Rosiny 1991; Barzel 1965).

Diese beiden zumeist getrennt voneinander verlaufenden Diskurse kreuzen sich in einer Reihe von Konzepten, die unter so illustren und schillernden Neologismen wie choreo-cinema, cinedance, ciné-choreography, film dance bzw. Filmtanz, Kamera-Choreographie, chorégrafilm, cameragraphy bis hin zum Videotanz die Annahme artikulieren, Film und Tanz könnten oder müssten eine besonders »innige Freundschaft«, so der Filmkritiker Konrad Karkosch (Karkosch 1952: o. S.), miteinander eingehen. Neben Karkosch haben sich seit den 1920er Jahren Tanz- sowie Film-Autoren zumeist in kürzeren Zeitschriftenartikeln und programmatischen Aufsätzen daran versucht, eine solche Fusionsästhetik von Film und Tanz einzufordern und zu entwerfen. Hier artikuliert sich ein Verhältnis von Film und Tanz, das in dem flottierenden Zwischenraum filmischer Abbildung >von « Tanz und der Selbstinszenierung der filmischen Mittel >als Tanz nicht mehr klar festzuzurren ist. Konzepte von Film- bis zum Videotanz, so meine ich, streben genau diese Doppelung an: Wie kann der gefilmte Tanz das Filmbild so affizieren, dass das bewegte Bild selbst einen Tanz produziert, einen Tanz, der ihm 
das bewegte Bild selbst einen Tanz produziert, einen Tanz, der ihm also zugleich innerlich und äußerlich ist? Und: Mit welchen Denkmodellen ist einem solchen unscharfen Verhältnis an der Schnittstelle von Film und Tanz noch theoretisch nachzukommen?

Damit widmet sich dieser Beitrag weniger den filmischen Umsetzungen einer solchen Interart-Ästhetik von Film und Tanz, wie sie beispielsweise im Musical- und Revuefilm, in experimentellen Filmpraktiken der 1940er bis 1960er Jahre oder auch im Videotanz seit Ende der 1970er Jahre realisiert worden sind. Vielmehr sollen hier aus filmtheoriegeschichtlicher Perspektive jene theoretischen Konzepte befragt werden, die jeweils auf eigene Art und Weise eine intermediale Poetik von Film und Tanz entwerfen und diskursivieren, somit zugleich deren Kritik-, Rezeptions- und Theoriegeschichte bilden. Die sehr heterogenen Konzepte von choreo-cinema, cinedance und Filmtanz möchte ich als ein vielstimmiges Diskursfeld oder, einem Konzept Laurent Guidos folgend, als einen »espace théorique« (Guido 2007: 11) untersuchen, in dem sich verschiedene Diskurslinien artikulieren, sich kreuzen, sich widersprechen, um anhand dieses Spannungsfeldes zentrale mediale Problematiken eines Zusammentreffens von Film und Tanz zu verhandeln. Das Diskursfeld um Begriffe wie choreo-cinema und cinedance scheint die mediale Verschränkung von Film und Tanz, so die Hypothese meines Beitrags, insbesondere über die Problematik der medialen Repräsentationsleistung des Films zu verhandeln. Wie ich im Folgenden anhand dreier Konzepte einer intermedialen Durchdringung von Film und Tanz aus den 1950er und 1960er Jahren freilegen möchte, scheinen sich solche Modelle wesentlich über die Infragestellung repräsentationalistischer Darstellungsverhältnisse sowie über deren Erweiterung durch performative Strategien einer tänzerischen Medialität zu konstituieren.

Wenn man das Verhältnis von Film und Tanz ausgehend vom sprachlichen Diskurs erkunden möchte, so ist dabei fortlaufend mitzureflektieren, dass sich eine solcherart geäußerte Interart-Poetik unter den spezifischen Bedingungen ihres sprachlich-begrifflichen Diskurses herausbildet. Dabei erweisen sich Begriffe wie »Tanz« oder »Choreographie« im Sprechen über Film als äußerst unzuverlässige Indikatoren für Tanz sim< Film. In seiner fast schon sprichwörtlich gewordenen NichtDiskursivität wird der Tanzbegriff in Filmtheorien und -kritiken nicht selten zu einer generalisierten Bezeichnung des Nicht-Benennbaren, dessen, was jenseits des Begrifflich-Verständlichen liegt. So beschreibt Jean-Paul Fargier den Tanzbegriff gewissermaßen als sprachliche »Notlösung« für den Filmkritiker: "Soudain dans un film, on ne sait plus comment nommer ce qui advient, sinon en disant que c'est de la danse« (Fargier 2005: 67). Filmtänzerische Konzepte bewegen sich von Anfang 
an in jenem aporetischen Spannungsfeld, welches ein Phänomen diskursiv zu erfassen versucht, das einen Grenzbereich exploriert, der mit sprachlich-begrifflichen Kategorien nicht einholbar ist. Damit verorten sich Konzepte wie choreo-cinema und Filmtanz in der Nähe von Momenten des subjektiven Kunsterlebens, »die gerade durch einen semantischen blinden Fleck, einen unaufgeklärten Bedeutungsrest faszinieren « (Bolz 1992: 20), sich über eine intuitive Rhetorik des Unsagbaren kommunizieren, wie das z.B. auch beim Aurabegriff Walter Benjamins oder dem punctum bei Roland Barthes der Fall sein mag. Interessanterweise reflektieren zahlreiche Autoren dieser theoriegeschichtlichen Diskurslinie den konstitutiven Charakter ihres Sprechens mit. So beginnen zahlreiche Texte mit der paradoxen Feststellung: eine intermediale Durchdringung filmischer und tänzerischer Prinzipien gebe es eigentlich (noch) nicht oder habe sich in der Filmpraxis noch nicht durchsetzen können. »The fact is that nobody has yet made a purely balletic film - the kind of film we think of when we consider the use of ciné-choreography « (Coton 1948: 31), schreibt beispielsweise der Kritiker A. V. Coton 1948. Und auch die Filmemacherin Maya Deren muss mit Blick auf die bisher realisierten Tanzfilme ernüchtert feststellen: »Das übliche unbefriedigende Resultat ist weder Fisch noch Fleisch - es ist weder guter Film noch guter Tanz« (Deren 1995: 88). Trotz des häufigen Vorkommens von Tanz im Film, welches die verschiedenen Autoren immer wieder durch einen inventarisierenden Blick auf eine Vielzahl von Filmbeispielen (vgl. z.B. Arroy 1926; Coton 1948; Karkosch 1983) oder durch einen Verweis auf entsprechende Tanzfilm-Kataloge (vgl. Martin 1946) zu belegen suchen, scheinen die Möglichkeiten einer intermedialen Verbindung von Film und Tanz, die den beiden Künsten seit Aufkommen des Kinematographen prophezeit wird (vgl. z.B. Tedesco 1923: 6), noch nicht ausgeschöpft. Durch diese Diskrepanz von filmtänzerischem Diskurs und seiner unzureichenden filmpraktischen Umsetzung, so die Sorge einiger Autoren, laufe wiederum ihr Sprechen Gefahr, als referenzloser Diskurs an Glaubwürdigkeit und Nachhaltigkeit zu verlieren. So befürchtet Coton, dass konventionelle Ballett-Filme wie THE RED SHOES (USA, 1948, Powell/Pressburger) sein Konzept cinéchoreographie als »merely a cliché in the mouths of film publicity writers « (Coton 1948: 30) diskreditieren könnten. Damit erkennen die Autoren nicht nur den performativen Charakter ihres Sprechens, sondern markieren die von ihnen entworfenen Modelle als einen theoretischen Möglichkeitsraum filmtänzerischer Praktiken, der sich vor deren filmischer Umsetzung artikuliert. Die Möglichkeit eines »avènement futur de la Danse véritable au cinéma« (Tedesco 1923: 10) wird visionär in die Zukunft projiziert; so betitelt z.B. Gardner Compton seinen Artikel bezeichnenderweise »Film Dance and Things to 
Come« (vgl. Compton 1968), wie auch der Untertitel von Cotons Artikel auf die »prospective use« (vgl. Coton 1948) seines ciné-choreographyKonzepts verweist.

\section{Choreo-cinema als Gegenentwurf zu Tanzfilmen mit dokumentarischer und archivarischen Funktion (Allegra Fuller Snyder)}

Wenn die manifestartigen Forderungen nach einer besonderen Verbindung filmischer und tänzerischer Darstellungsmittel vor allem als Gegenentwurf zu bestehenden Praktiken der Tanzverfilmungen artikuliert werden, so scheinen sich diese vor allem gegen >rein reproduzierende Aufzeichnungen eines originären Tanzereignisses mit dokumentarischer und archivarischer Funktion zu richten. Eine solche Gegenüberstellung wird beispielsweise in der Typologisierung verschiedener Arten von Tanzfilmen deutlich, die die Tanzwissenschaftlerin Allegra Fuller Snyder im Jahr 1965 vorstellt und die bis heute eine gewisse Gültigkeit (beispielsweise für die Kategorisierung bei Tanzfilm-Festivals, vgl. Rosiny 1999) bewahrt hat. In ihrem Artikel »Three kinds of Dance Films« in der US-amerikanischen Zeitschrift »Dance Magazine« unterscheidet Snyder je nach intendiertem Verwendungszweck der Filmaufnahmen drei verschiedene Arten von Tanzfilmen. Die erste Kategorie, der Notationsfilm, hat für Snyder eine mnemotechnische, den schriftlichen Notationssystemen von Tanz vergleichbare Speicherfunktion: mit der Filmkamera wird die Choreographie möglichst präzise und vollständig aufgenommen, um so spätere Analysen und Rekonstruktionen der Bewegungsabfolgen zu ermöglichen. Als zweite Kategorie differenziert Snyder den documentary film - im Sinne einer Bühnenaufzeichnung -, der eine im Theaterdispositiv stattfindende Tanzaufführung an die Mit- und Nachwelt weitergeben soll. Zweck der filmischen Aufzeichnung ist hier »to capture the essence of the choreographer's intend, to document the work as it is performed in order to build our literature and carry these works of art to the student or the public, present or future « (Snyder 1965: 39). Während die ersten beiden Kategorien von Tanzfilmen das Zusammentreffen von Medientechnologie und menschlichem Körper unter dem Paradigma des Mediums als Speicher- und Übertragungstechnik verhandeln, entwirft Synder unter dem Neologismus choreo-cinema - einer offensichtlichen Kombination der Wörter choreography und cinema - eine mediale Funktion des tänzerischen Films, die nicht mehr als sekundäre Aufzeichnung der Darstellung eines profilmischen Tanzereignisses verpflichtet ist. Vielmehr kon- 
zipiert Snyder die Fusion von Film und Tanz als einen Ort, an dem ein schöpferisches Potenzial freigesetzt wird, das die jeweiligen Möglichkeiten der Einzelmedien transzendiert: »In choreo-cinema the film-maker and the choreographer work together to create something which could not exist without the fusion of the two arts« (Snyder 1965: 34).

Mit der Gegenüberstellung der reproduktiven Funktionen des Filmmediums in den Notationsfilmen und Bühnenaufzeichnungen und den in choreo-cinema angedeuteten schöpferischen Möglichkeiten des Films spiegelt Snyders Tanzfilm-Typologie die zentrale Debatte klassischer Filmtheorien wider, wie sie auch Jerome Delamater in der Begegnung von Film und Tanz angelegt sieht: »The problems inherent in filmed dance are, in a sense, a microcosm of the disagreements among film theorists. Film is a re-producing agent according to Siegfried Kracauer and André Bazin; film is primarily a manipulating medium accroding to Rudolf Arnheim, Béla Balázs, Sergei Eisenstein, and others.« (Delamater 1981: 5)

Insofern Allegra Fuller Snyder die Unterscheidung von choreocinema und repräsentationalen Tanzverfilmungen unterschiedlichen Gebrauchshorizonten zuschreibt (vgl. Snyder 1965: 39) bzw. als rein stilistische Differenz verhandelt, können reproduzierende sowie künstlerische Funktionen der Tanzfilme vergleichsweise konfliktlos in ihrer Typologie koexistieren. Als unterschiedliche mediale Inszenierungen sind die verschiedenen filmischen Bearbeitungsmöglichkeiten in Snyders Typologie gegeneinander austauschbar und können als Derivate desselben originären Tanzes drei mögliche filmische Variationen darstellen: »all three kinds of film could be made of the same dance« (Snyder 1965: 39).

\section{Tanz im Zeitalter seiner technischen Reproduzierbarkeit}

Problematischer wird die Frage nach der Möglichkeit der Tanzverfilmung bei Autoren, die die Trennlinie von repräsentationalen Tanzverfilmungen und ihren Forderungen nach einer Fusionsästhetik von Film und Tanz nicht als formale, sondern als medienontologische Differenz konzipieren und von einer wesentlichen Unvereinbarkeit von Film und Tanz ausgehen: »There seems to exist a basic contradiction between the two plastic arts of stage and and screen, which cannot be solved by technique only.« fasst Agnes Bleier-Brody (Bleier-Brody 1968) die Haltungen verschiedener Autoren des Tanz- und Filmbereichs für den UNESCOKatalog zusammen. 
Damit scheinen Forderungen nach einer intermedialen Ästhetik von Film und Tanz auch den Topos einer Entgegensetzung von Aufführung und Aufzeichnung aufzurufen, wie ihn Gabriele Brandstetter diagnostiziert. In einer Diskurstradition, die den Tanz als »essentialisierte Form für das Ephemere schlechthin reklamiert«, beobachtet Brandstetter (Brandstetter 2005: 203) eine »Emphatisierung von Aufführung, der gegenüber die Aufzeichnung sekundär ist« (Brandstetter 2005: 199), eine Hierarchisierung des ephemeren Bühnenereignisses gegenüber seiner medial reproduzierten Festschreibung. Vor dem Hintergrund eines Tanzverständnisses, das sich wesentlich über die präsentische Leibhaftigkeit des Tänzers sowie über das Aura generierende Theaterdispositiv konstituiert, gerät die filmische Aufzeichnung von Tanz in den quasi-moralischen Verdacht, eine wesenhafte Transformation am Tanz vorzunehmen. Dies veranschaulichen neben der konsequenten Weigerung einiger berühmter Choreographen wie Isadora Duncan und Vaslav Nijinsky, ihre Tänze der Kamera preiszugeben, beispielsweise auch die erschütterten Kommentare zu den Filmaufnahmen der berühmten Ballerina Anna Pawlowa aus den 1920er Jahren, die nicht einmal einen Bruchteil der auratischen Bühnenpräsenz der legendären Tänzerin einzufangen vermögen, schlimmer noch: dem Bühnendispositiv entrissen, wirkt die grande dame des Balletts geradezu lächerlich: »[...] audiences unfamiliar with the art of ballet laugh at these films, at the jerky images of the lady with the big feet standing on pointe, pulling the spetals represent a California puppy« (Turnbaugh 1970: 18). Gerade die technischen Medien, deren Bilder sich ohne menschlichen Eingriff gleichsam »selbst« zu produzieren scheinen, stehen für Tänzer und Choreographen unter dem ontologisierten Verdacht, die Übertragung des Tanzes nur unvollständig, als Substraktion oder gar Negation des Tanzes leisten zu können. So »stellen Fotografie und Film als künstliche Medien das Naturhafte, Authentische des Tanzes und als Reproduktionsmedium die Einzigartigkeit des tänzerischen Originals in Frage« (Klein 2000: 7). Die Erkenntnis, dass die technische Reproduktion eines Kunstwerkes dieses einer qualitativen Transformation seines »innersten Kerns« aussetze, verhandelt Walter Benjamin in seinem Kunstwerk-Aufsatz von 1936 (vgl. Benjamin 1963: 23) über eine Reihe dichotomischer Gegenüberstellungen von Original und Reproduktion, Dauer und Flüchtigkeit, Ferne und Nähe. Auch in Benjamins Denkmodell führt die filmische Reproduktion als Herauslösung des Tanzes aus dem Aura generierenden Theaterdispositiv (vgl. Benjamin 1963: 12) sowie die Ablösung des Tanzes von der Leibhaftigkeit des Tänzers (vgl. Benjamin 1963: 27) gleichsam sautomatisch $<\mathrm{zu}$ einer Entauratisierung des aufgeführten Tanzes. Aber schon bei Benjamin klingt die Möglichkeit an, den Verfall der Aura nicht 
als endgültigen Verlust zu betrachten. Neben externen Aura-Zuschreibung über den Starkult (vgl. Benjamin 1963: 28) und die sakralisierende Rhetorik der frühen Filmtheorie (vgl. Benjamin 1963: 23) könnten die neuen kinematographischen Bilder Benjamin zufolge eigene ästhetische Effekte wie z.B. den Chock produzieren (vgl. Benjamin 1963: 39; vgl. z.B. auch Lehmann 2003).

Vor dem Hintergrund dieser hier nur flüchtig skizzierten Lesart von Benjamins Annahme einer möglichen Reauratisierung mit den Mitteln der Reproduktionstechnik, lassen sich auch Konzepte wie choreo-cinema und film dance als auf den ersten Blick aporetische Versuche lesen, dem durch die filmische Reproduktion herbeigeführten Auraverlust des Tanzes durch filmische Mittel (wiederum) entgegenzuwirken. »Es ginge darum«, so formuliert Gabriele Brandstetter ganz ähnlich,

»einen solchen Versuch einer Kunst der Aufzeichnung als ein paradoxes Unternehmen aufzufassen und in Gang zu setzen: [...] eine Transkription, die auch zu erkennen gibt, dass eben dieser gestaffelte Prozess der Nachträglichkeit in der Aufzeichnung nicht etwa einen Verlust des immer schon Vergangenen, Ephemeren der Aufführung kompensiert, sondern diesem etwas (zurück)gibt! « (Brandstetter 2005: 206-207)

Damit sich Tanz und Film zu einer solchen saufführenden Aufzeichnung übereinander schieben können, ohne sich dabei in der Paradoxie einer Gleichzeitigkeit des Gegensätzlichen zu paralysieren, müssen filmtänzerische Konzepte hierarchisch gedachte Prämissen zum Verhältnis von Original und Reproduktion bzw. Aufführung und Aufzeichnung umwälzen und in eine dynamische Konstellation überführen. Anhand der filmtänzerischen Konzepte von Arthur Maria Rabenalt und Maya Deren möchte ich im Folgenden exemplarisch skizzieren, welche konzeptuellen Bemühungen diese Autoren an der Schnittstelle von Film und Tanz unternehmen, um in ihren Theorien ein Modell filmischer Medialität zu konturieren, das den Film nicht einseitig auf seine reproduzierenden oder transformierend-schöpferischen Funktionen reduziert, sondern vielmehr eine Überblendung aufführender und aufzeichnender Strategien betont. 


\section{Von tanzenden Kameras und Filmchoreographien: \\ Filmischer Tanz als Verdopplungsstrategie (Arthur Maria Rabenalt)}

In seinem 1960 erschienen Buch TANZ UND FILM widmet sich der österreichische Regisseur Arthur Maria Rabenalt ausführlich der Frage, wie »die Kunst des Filmes mit der Kunst des Tanzes legitim aber leidenschaftlich zu vermählen« (Rabenalt 1960: 15) sei. Wie viele andere Autoren entwickelt Rabenalt sein Modell einer filmtänzerischen InterartPoetik als einen spezifischen Lösungsversuch für die als problematisch wahrgenommene Reproduzierbarkeit des Tanzes durch den Film. Dabei geht er von der Erkenntnis aus, »daß [Tanz] im Film etwas anderes sein müßte als Tanz auf der Bühne: und zwar bereits von der choreographischen Idee und grundsätzlichen Intentionierung her« (Rabenalt 1960: 26). Im Gegensatz zu einem rein aufzeichnenden Filmgebrauch fordert Rabenalt eine explizite >Filmisierung des Tanzes, nämlich »die freie souveräne Übertragung einer Kunstform in das Medium des Films« (Rabenalt 1960: 14). Der gefilmte Tanz müsse eine »äußere und innere Umwandlung « über sich ergehen lassen, »wenn er in das Medium des Films eingehen und zur neuen Ausdrucksform des filmischen Tanzes werden soll« (Rabenalt 1960: 18). So untersucht Rabenalt unter der Fragestellung »Welches sind nun im Einzelnen die speziellen Ausdrucksmittel, mit denen der Film ein tänzerisches Geschehen zum filmeigenen Tanz macht? (Rabenalt 1960: 20) systematisch und detailliert die Möglichkeiten von Kamerabewegung, filmischen Raumkonstruktionen und Montageprinzipien für die >Filmisierung « des dargestellten Tanzes.

Die Bewegtheit der Kamera konstituiert für Rabenalt dabei ein zentrales Mittel, um den gefilmten Tanz in filmischen Tanz zu überführen:

"Auf alle Fälle musiziert die Filmkamera mit, in gleichem Takt oder in kunstgewollten, absichtsvollen Synkopen. Der sich bewegende Aufnahmeapparat fügt aber auch der tänzerischen Bewegung sein eigenes Bewegungsmoment bei. Er bewegt sich im Sinne der filmischen Gestaltungsabsicht innerhalb der tänzerischen Bewegung - er tanzt mit.« (Rabenalt 1960: 19)

Die auch bei vielen anderen Autoren des filmtänzerischen Diskursfeldes prominente Idee der >tanzenden Kamera (vgl. auch Karkosch 1956; Tyler 1967) rekurriert auf die Annahme einer grundlegenden Analogie von Tänzer und Kamera, die nicht nur als zwei bewegte Körper im gleichen Raum agieren, sondern deren Bewegungen aus der jeweiligen Verdoppelung von Bewegungskörper und Wahrnehmungskörper in ein Interdependenz-Verhältnis zueinander geraten. Der Bewegungseffekt sei immer 
ein Zusammenwirken, das aus der Beziehung von filmischer und tänzerischer Bewegungsproduktion und -wahrnehmung entstünde und sich gleichsam kinästhetisch auf den Zuschauer übertragen könne, »damit der Zuschauer in das Tanzerlebnis einbezogen und sein rein betrachtender Zustand aufgehoben wird « (Rabenalt 1960: 19). In Rabenalts Annahmen zur Doppelfunktion der Kamera ist die Ambivalenz reproduktiver und schöpferischer Anteile bereits angelegt, wenn er schreibt: „Die Kamera ist nicht mehr nur phototechnisches Aufzeichnungs- und Konservierungsmittel, sondern wirkt selber mit, >stellt dar« und zwar in prominenter Form als gestaltende, autonome neue Energie.« (Rabenalt 1960: 20)

Auch über das filmische Montageprinzip sei gefilmter Tanz in filmischen Tanz zu überführen, da die Montage das endgültige Anordnen der Bewegungsabschnitte, und damit den eigentlichen Akt des Choreographierens übernehme. Sie lenke nicht nur den Blick des Zuschauers auf den Tanz, sondern wirke als strukturierendes Zusammenfügen von tänzerischen Bewegungsmomenten selbst wie eine »zweite Choreographie«, die den gezeigten Tanz verdoppeln und intensivieren könne, so dass die 〉Filmisierung des dargestellten Tanzes zu einem Gemeinschaftswerk des Tanzes und der Gesamtheit der filmischen Mittel werde:

»Zur Choreographie der Tanzinszenierung gesellt sich gleichwertig die Choreographie des regieplanmäßig geführten Aufnahmegerätes; dem einstudierten Musik- und Bewegungsrhythmus der Tänzer koordiniert sich der künstlerisch beabsichtigte Bildrhythmus der Montage. Beide sind nicht nur künstlerische Ausdrucksmittel des Tanzes im Film, sondern gleichgestellte Partner im vermeintlichen Solo, Pas des deux oder im großen Ensemble des tänzerischen Vorganges.« (Rabenalt 1960: 20)

Konzipiert Rabenalt das Zusammentreffen von Film und Tanz zunächst als scheinbar systematische Überführung eines abgefilmten Tanzes in einen genuin filmischen Tanz, betont er über die Metapher des tänzerischen Duos zunehmend die Verdoppelung der Aufzeichnungs- und Repräsentationsfunktionen des Films über dessen Potenzial, als »mitbestimmender, mitgestaltender Partner« (Rabenalt 1960: 27) in den Produktionsprozess des filmischen Tanzes einzugreifen. So sind die vom Film produzierten tänzerischen Effekte Rabenalt zufolge nicht mehr eindeutig auf filmische oder tänzerische Bewegungsstrategien zurückzuführen, sondern nur noch unscharf als >Zusammenspiel filmischer und tänzerischer Strategien zu erfahren. Der konzeptuelle >Kunstgriffı in Rabenalts Modell eines filmischen Tanzes besteht also vor allem darin, dass er die filmische Überformung des Tanzes nicht als dem Tanz äußerliche Kompensationsstrategie annimmt, sondern den Einsatz der filmischen Mittel wiederum aufs Engste an tänzerische Prinzipien rückbindet. So >naturali- 
siert< Rabenalt den Einsatz der filmischen Mittel gleichsam, wenn er sich in beinahe wagnerianischem Pathos auf die Annahme einer ontologischen Nähe von Film- und Tanzkunst beruft. Tanz und Film seien »wie ungleiche und altersmäßig durch Jahrhunderte getrennte Töchter des selben Ursprunges: des unsterblichen MIMUS!« (Rabenalt 1960: 5) Filmtänzerische Momente als Evidenzwerdung dieser ursprünglichen Verwandtschaft der darstellenden Künste stellen sich Rabenalt zufolge dann ein, wenn der Einsatz der filmischen Mittel durch den dargestellten Tanz affiziert und motiviert sei und so mit diesem »mittanzen und selbst ein entscheidender Solist des tänzerischen Geschehens werden kann« (Rabenalt 1960: 14). Indem Rabenalt den Einsatz von Montage und Kamerabewegungen diskursiv als tänzerische Strategien inszeniert, verschalten sich filmische und tänzerische Bewegungsebenen in seinem FilmtanzKonzept gleichsam zirkulärkausal miteinander.

Über die von Rabenalt wiederholt argumentierte Äquivalenzannahme von Tänzer und Kamera, sowie von Choreographie und Montage, wird somit ein Zeichenmodell entworfen, in dem sich die visuelle Repräsentation oder (im semiotischen Vokabular) die ikonischen Ähnlichkeitsbeziehungen und die indexikalische Einschreibung des Tanzes in das Filmbild durch die Eigenbewegung des Films in Kamerabewegung und Montage $\mathrm{zu}$ verdoppeln scheinen. Der Film kommuniziert nicht mehr nur über die visuelle Referentialität seines photographischen Bildes, sondern Montage und Kamerabewegungen werden zu deren komplementären Darstellungsstrategien. Im filmtänzerischen >Duo< von Film und Tanz kündigt sich damit ein Darstellungsverhältnis an, das nicht mehr auf der Nachgestelltheit der Re-präsentation hinter dem originären, aufgezeichneten Tanz beruht, sondern das Bezeichnende und das Bezeichnete in eine Beziehung der Gleichzeitigkeit und simultanen Wahrnehmbarkeit versetzt.

\section{Zwischen photographischem Realitätszugriff und filmischer Inszenierung: Film dance als Erfahrung des Tänzerischen (Maya Deren)}

Auch die amerikanische Filmemacherin Maya Deren verhandelt in ihrem Konzept von film dance auf zentrale Art und Weise die konzeptuelle Vereinbarkeit von repräsentationalen und performativen Darstellungsstrategien am Schnittpunkt von Film und Tanz. Parallel zu ihren filmischen Experimenten mit Tänzern der Dunham Company hinterfragt sie ab Mitte der 1940er Jahre in zahlreichen Artikeln, Essays und Vorträgen 
die »Möglichkeiten, filmisch eine Form für den Tanz zu finden, in der Choreographie und Bewegung präzise auf die Eigenschaften der Kamera, ihre Beweglichkeit beispielsweise, hin konzipiert wären« (Deren 1995: 88). Dass der Tanz als »Choreography for Camera« - in Anlehnung an ihren wohl bekanntesten Tanzfilm A STUDY OF CHOREOGRAPHY FOR CAMERA (Deren 1945) - für seine Reproduzierbarkeit konzipiert sein müsse, ist für Deren anders als für Rabenalt kein spezifisches Problem der Verfilmung von Tanz; vielmehr bestehe die Repräsentationsproblematik des Film grundsätzlich darin - so Deren in der Rhetorik ihrer essentialistisch-orientierten Filmtheorie (vgl. Jackson 2001) -, ihm äußerliche Elemente in seinen filmspezifischen Darstellungsmodus zu überführen:

"The form proper of film is [...] accomplished only when the elements, whatever their original context, are related according to the special character of the instrument of film itself - the camera and the editing - so that the reality which emerges is a new one - one which only film can achieve and which could not be accomplished by the exercize [sic] of any other instrument.« (Deren 2001: 40)

Vor allem in ihrem Artikel »Kamera-Arbeit: Der schöpferische Umgang mit der Realität« (1960) begründet Deren ihre Filmtheorie auf dem besonderen Realitätsstatus des photographischen Bildes, dem die »objektive Autorität des Realen« (Deren 1995: 64) anhafte. Über die reine Ähnlichkeitsbeziehung des Bildes hinaus bilde das photographische Verfahren einen »geschlossenen Kreis« (Deren 1995: 55) zwischen dem Aufnahmeapparat und dem gefilmten Gegenstand, welcher sich gleichsam selbst ins photographische Bild einschreibe: Die Photographie sei »ein Verfahren, durch das ein Gegenstand mit Hilfe von Licht oder lichtempfindlichen Material sein eigenes Bild produziert« (Deren 1995: 55). In Analogie der Idee einer »émanation du référent«, mit der Roland Barthes die von Deren formulierten Überlegungen zum Status des photographischen Bildes 1980 in »La chambre claire« (Barthes 1980: 126) auf den Punkt bringen sollte, sei das photographische Bild laut Deren immer auch eine Authentifizierung des Dargestellten. Aufgrund seiner gleichsam physischen Beziehung zur Realität, so Deren, »muss ein Photo [...] als eine Form der Realität selbst begriffen werden« (Deren 1995: 57).

Durch die Montage verfüge der Film jedoch zugleich über die Möglichkeit, auf diese »Form der Realität« gestaltend zuzugreifen und durch die Anordnung der Filmbilder eine neue Realität zu erschaffen:

»Erst die filmische Montage bringt die Folgebeziehungen der Bilder zueinander hervor, die ihnen, je nach ihrer Funktion, eine besondere oder neue Bedeutung 
verleiht; sie schafft einen Kontext, eine Form, die die Bilder verändert, ohne jedoch ihre Aspekte zu verzerren, ihre Realität und Autorität zu mindern oder jene Vielfalt potentieller Funktionen zu verringern, die für alles Reale charakteristisch ist.«(Deren 1995: 65)

Die Montage führt jene Differenz zwischen dem photographischen Bild und seinem Referenten ein, die die Konstruktion einer filmischen Welt und deren Illusionsbildung voraussetzt. Im Gegensatz zur theatralen Kommunikation, die auf der Ko-Präsenz von Zuschauer und Darsteller beruht, konstituiere sich die filmische »Wirklichkeit« gerade über die Trennung und Distanz von einer als außerfilmisch angenommenen Realität, so Deren: »In mancher Hinsicht kann gerade sogar das Fehlen der physischen Präsenz des Schauspielers, die im Theater so wichtig ist, im Film unser Realitätsgefühl verstärken. Wir können zum Beispiel an die Existenz des Monsters glauben, solange man nicht von uns verlangt zu glauben, daß es im gleichen Raum mit uns ist.« (Deren 1995: 59)

Bettet die Montage das photographische Bild in neue sinnbildende Zusammenhänge, so überträgt das photographische Bild als Grundelement des Films wiederum seine Autorität des »Selbst-Gesehenen « (Deren 1995: 36) auf die phantastischen Vorstellungen, die der entfremdende Eingriff zu produzieren vermag. Erst über diese gegenseitige Übertragung von Realitätsbezug und den gestaltenden filmischen Eingriffen kann es dem Film gelingen, so Deren, ihm äußerliche Elemente wie den Tanz in seinen Darstellungsmodus zu integrieren. So besteht die Ontologie des Films Deren zufolge in einem Oszillieren zwischen dem transparenten Realitätsbezug des photographischen Bildes und der entfremdenden Gestaltung der filmischen Inszenierung. Diesen für ihr Konzept von film dance konstitutiven Balanceakt beschreibt Deren auch als Methode des »kontrollierten Zufalls«:

»Unter >kontrolliertem Zufalk verstehe ich das Einhalten eines empfindlichen Gleichgewichts zwischen dem, was spontan und natürlich gegeben ist, um von der unabhängigen Existenz des Tatsächlichen zu zeugen, und den Personen und Begebenheiten, die absichtlich in Szene gesetzt werden. [...] Das fiktionale Ereignis, das dann abläuft, ist zwar selbst künstlerischer Art, borgt sich jedoch Realität von der realen Szenerie.« (Deren 1995: 60)

Indem Derens Filmtheorie die filmischen Tendenzen von Wirklichkeitsbezug und Fiktionalisierung aufs Engste miteinander verzahnt, treibt sie die Dichotomie von Aufführung und Aufzeichnung, von Original und Reproduktion in eine Inversion. Insofern der gefilmte Tanz einerseits durch den Realitätszugriff des photographischen Bildes bewahrt und zugleich filmisch transformiert wird, verdoppelt sich ihr Konzept von 
Filmtanz zu einer filmisch entfremdeten Tanz-Aufzeichnung oder (anders herum gesehen) zu einer filmischen Konstruktion von Tanz, die sich über das photographische Bild wiederum naturalisiert. Um die filmtänzerischen Effekte weder einseitig auf den dargestellten Tanz und die photographische Referentialität, noch auf seine filmische Dynamisierung und Entfremdung zurückführen zu müssen, ersetzt Deren die Dichotomie des Films zwischen Reproduktion und Produktion durch den Begriff der 〉Erfahrung〈. Der Film, so Maya Deren, müsse »eine totale Erfahrung schaffen, die dem eigentlichen Wesen dieser Kunstform so sehr entspringt, dass sie von seinen Mitteln nicht zu trennen ist« (Deren 1995: 70). Das schwindelerregende Kreiseln zwischen photographischem Realitätszugriff und filmisch-ästhetischer Fiktionalisierung ergreift in Derens film dance-Konzept den gefilmten Tanz, der als >Erfahrung des Tänzerischen in eine rezeptive Kategorie jenseits der repräsentationalistischen Logik überführt wird, wo Darstellendes und Dargestelltes, Aufführung und Aufzeichnung, Original und Reproduktion nicht mehr trennscharf voneinander zu unterscheiden sind, sondern sich die tänzerischen Effekte durch deren chiastische Verschränkung produzieren.

\section{Ausblick: Filmtanz als Modell eines gestischen Films?}

Die hier skizzierten Positionen von Snyder, Rabenalt und Deren deuten exemplarisch an, wie der Diskurs um filmtänzerische Konzepte nicht nur die Frage der filmischen Darstellbarkeit von Tanz im Film verhandelt, sondern an der Schnittstelle von Film und Tanz komplexe Darstellungsmodelle entwirft, in denen die hierarchischen Dichotomien von Aufführung und Aufzeichnung, Original und Reproduktion in eine Konstellation dynamischer Wechselwirkungen überführt werden.

Als Gegenentwurf zu Tanzfilmen mit dokumentarischer Intention wird die intermediale Durchdringung und Film und Tanz bei den hier untersuchten Autoren als eine konzeptuelle Verschränkung repräsentationaler und performativer Darstellungsstrategien konzipiert, die mal als Nebeneinander verschiedener stilistischer Varianten (Snyder), mal als reauratisierende Verdoppelung (Rabenalt) bzw. chiatisches Ineinander (Deren) in einem medialen Modell von Filmtanz integriert werden. Damit findet eine Überblendung jener beiden Funktionen statt, die in der Filmtheoriegeschichte zumeist als antagonistische Positionen verhandelt werden: die Abbildungsfunktion des Films sowie die filmische Möglichkeit, tänzerische Effekte zu produzieren, verdoppeln und überlagern sich, blenden sich zeitweise gegenseitig aus. 
Indem diese filmtänzerischen Konzepte die beiden Variablen Film und Tanz in ein unscharfes Verhältnis von Gleichzeitigkeit und Verdoppelung setzen, entziehen sie sich Erklärungsmodellen einer repräsentationalen Logik von Substitution, Vergegenwärtigung und Referenz (vgl. Mersch 2002: $210 \mathrm{ff}$.), und lassen ganz im Sinne von Deleuzes Filmtheorie das Potenzial des filmischen image-mouvement zum Bruch mit einem repräsentationalen Bildverständis aufscheinen. In den durch diese Überlagerungen entstehenden Zeichen- oder Medienmodellen tritt die referentielle Funktion des Filmbildes mitunter hinter jenen komplementären Strategien zurück, die man im Anschluss und in Erweiterung an Villém Flusser (vgl. Flusser 1991) vielleicht als `Gesten des Films« oder mit Erika Fischer-Lichte (vgl. Fischer-Lichte 2004) als >performative` Strategien des Films bezeichnen könnte: Schnitt und Montage, Kamerabewegung und der Zoom durch verschiedene Einstellungsgrößen produzieren und performieren eigene `tänzerischeく Bewegungen, die sich nicht mehr in eine referentielle filmische Sinnstiftung einordnen lassen, sondern diffuse Effekte des Tänzerischen generieren.

So vermerkt auch Giorgio Agamben in Anschluss an Deleuzes Diagnose einer nicht-(nur)-repräsentationalen Ordnung filmischer Bilder: »Das Element des Kinos ist die Geste, und nicht das Bild (Agamben 2004: 42). Unter dem Begriff der Geste versteht Agamben bekanntermaBen nicht nur eine Körperbewegung wie den Tanz, sondern eine spezifische Form der Medialität, in der sich die Verweisbewegung auf einen äußerlichen Referenten nur noch andeutet, eine Verweisbewegung, die zwischen Potenz und Akt, Mittel und Zweck oszilliert. Auch in Rabenalts und Derens Konzepten scheint der referentielle Verweis auf einen außerfilmischen Tanz nicht mehr vollständig ausgeführt zu werden, sondern nur noch Aufführung einer (möglichen) Handlung, in Agambens Sinne also »die Darbietung der Mittelbarkeit, das Sichtbar-Werden des Mittels als einem solchen « (Agamben 2004: 44) zu versprechen. Im Oszillieren zwischen referentiellen und performativen Ebenen der filmischen (Re)Produktion von Tanz könnte schließlich das Medienmodell eines gestischen Films im Sinne Agambens angelegt sein, das - vom tänzerischen Moment affiziert - jenen Bedeutungsüberschuss produziert, dem sprachlich nur noch mit der begrifflichen Kategorie »Tanz« nachzukommen ist. Am Fluchtpunkt einer solchen Auffassung von Filmtanz als Modell eines gestischen Films würde sich schließlich die Möglichkeit eines »Tanzfilms ohne Tänzer« (vgl. Knight 1967) abzeichnen, die sich in die gleichermaßen furcht- und lustbesetzte Utopie einer Ablösung des Tanzes vom menschlichen Körper einschreibt, wie sie Jean Genet 1949 in Adame Miroir (zit. n. Brandstetter 1993: 411) heraufbeschwört, wenn er schreibt: »So wird es schließlich Dein Bild sein, das für Dich tanzt«. 


\section{Literatur}

Agamben, Giorgio (2004): »Noten zur Geste«. In: Hemma Schmutz/ Tanja Widman (Hg.), Dass die Körper sprechen, auch das wissen wir seit langem, Köln: Walther König, S. 39-48.

Arroy, Juan (1926): »Danses \& Danseurs de Cinéma«. Cinémagazine, Nr. 48, November 1926, S. 427-430.

Barthes, Roland (1980): La chambre claire. Notes sur la photographie, Paris: Cahiers du Cinéma, Gallimard, Seuil.

Barzel, Ann (1965): »Films for Remembrance«. Dance Magazine, September 1965, S. 22-26.

Benjamin, Walter (1963): Das Kunstwerk im Zeitalter seiner technischen Reproduzierbarkeit, Frankfurt am Main: Suhrkamp.

Bleier-Brody, Agnes (1968): »The dancing screen«. In: UNESCO (Hg.),

Ten years of films on ballet and classical dance 1956-65, Paris: UNESCO, o. S.

Bolz, Norbert (1992): »Walter Benjamins Ästhetik«. In: Uwe Steiner (Hg.), Walter Benjamin, 1892-1940, zum 100. Geburtstag, Bern: Lang, S. 11-32.

Brandstetter, Gabriele (Hg.) (1993): Aufforderung zum Tanz. Geschichte und Gedichte, Stuttgart: Reclam.

Brandstetter, Gabriele (2005): Bild-Sprung. TanzTheaterBewegung im Wechsel der Medien, Berlin: Theater der Zeit.

Brooks, Virginia/Rosiny, Claudia (1991): »Tanz konservieren. Über die Kunst, Tanz im bewegten Bild zu dokumentieren«. Tanzdrama, Nr. 15, S. 4-6.

Compton, Gardner (1968): »Film Dance and Things to Come«. Dance Magazine, Januar 1968, New York, S. 34-37.

Coton, A.V. (1948): »Ciné-Choreography. Some notes on its prospective use «. Ballet and Opera, London, Vol. 6, Nr. 1, S. 30-37.

Delamater, Jerome (1981): Dance in the Hollywood Musical, Ann Arbour: UMI Research.

Deren, Maya (1995): Choreographie für eine Kamera - Schriften zum Film, hrsg. von Jutte Hercher, Ute Holl, Kathrin Reichel, Kira Stein, Petra Wolff, Hamburg: Material-Verlag.

Deren, Maya (2001): »Anagram of Ideas on Art, Form and Film«. In: Bill Nichols (Hg.), Maya Deren and the American Avant-Garde, Berkeley, Los Angeles, London: University of California Press, S. 267-322.

Fargier, Jean-Paul (2005): »La danse nuptiale des genres polygames«. Vertigo. Esthétique et Histoire du Cinéma, Hors série »Danses«, Oktober 2005, Paris, S. 67-69. 
Fischer-Lichte, Erika (2004): Ästhetik des Performativen, Frankfurt/M.: Suhrkamp.

Flusser, Vilém (1991): Gesten. Versuch einer Phänomenologie, Düsseldorf, Bensheim: Bollmann.

Guido, Laurent (2007): L'Age du rythme. Cinéma, musicalité et culture du corps dans les théories francaises des années 1910-1930, Lausanne: Payot.

Jackson, Renata (2001): »The Modernist Poetics of Maya Deren«. In: Bill Nichols (Hg.), Maya Deren and the American Avant-Garde, Berkeley, Los Angeles, London: University of California Press, S. 47-76.

Jordan, Stephanie/Allen, Dave (Hg.) (1993): Parallel Lines. Media Representations of Dance, London: The Arts Council of Britain \& John Libbey \& Co.

Karkosch, Konrad (1952): »Die entfesselte Bewegung. Der Tanz erobert den Film«. Filmforum, März 1952, o. S.

Karkosch, Konrad (1956): »Film und Tanz. Verfilmter Tanz. Filmischer Tanz. Absoluter Tanz«. Filmjournal, 11.10.1956, o. S.

Karkosch, Konrad (1983): »Film und Tanz. Vom `gefilmten Tanz« zum >Filmtanz«. Ballett-Journal - Das Tanzarchiv, 31. Jahrgang, 1/83, S. 8-20.

Klein, Gabriele (2000): »Tanz \& Medien: Un/heimliche Allianzen«. In: Dies. (Hg.), Tanz Bild Medien, Hamburg: LIT, S. 7-18.

Knight, Arthur (1967): »Cinedance«. Dance Perspectives, Nr. 30, S. 4-9.

Krah, Hans (2003): »Tanz-Einstellungen. Ein Blick auf die Geschichte des Tanzes im Film«. In: Ernst W.B. Hess-Lüttich (Hg.), TanzZeichen. Vom Gedächtnis der Bewegung, Kodikas/Code, Ars Semeiotica, An international Journal of Semiotics, Vol. 26, Nr. 3-4, S. 251-271.

Lehmann, Hans-Thies (2003): »Theater, Aura, Chock und Film«. In: Harald Hillgärtner/Thomas Küpper (Hg.), Medien und Ästhetik, Bielefeld: Transcript, S. 69-82.

Martin, John (1946): The Dance - The Story of the Dance told in pictures and text, New York: Tudor Publishing Company.

Mersch, Dieter (2002): Was sich zeigt. Materialität, Präsenz, Ereignis, München: Fink.

Rabenalt, Arthur Maria (1960): Tanz und Film, Berlin: Rembrandt Verlag.

Rosiny, Claudia (1999): Videotanz. Panorama einer intermedialen Kunstform, Zürich: Chronos Verlag.

Snyder, Allegra Fuller (1965): »Three kinds of Dance Films«. Dance Magazine, September 1965, S. 34-39. 
Tedesco, Jean (1923): »La danse sur l'écran«. Cinéa-Ciné pour tous, Nr. 1,15 novembre 1923 , S. 6-11.

Turnbaugh, Douglas Blair (1970): »Cinema, Dance \& Cine-Dance«. Filmmaker's Newsletter, November 1970, S. 14-24.

Tyler, Parker (1967): »Cine-dance«. Dance Perspectives, Nr. 30, S. 14 f. 
